\title{
New Generator Design with Enhanced Output Waveform for Non- Destructive Radar Sensor Measurements
}

\author{
Younes Ahajjam ${ }^{1,3 a}$, Otman Aghzout ${ }^{1,2}$, José M. Catala-Civera ${ }^{3}$, Felipe Peñaranda-Foix ${ }^{3}$ and Abdellah Driouach ${ }^{1}$ \\ ${ }^{1}$ Laboratory of Communications and Systems, Faculty of Sciences, Abdelmalek Essaadi University, Tetouan, Morocco \\ ${ }^{2}$ Electronics and Microwave Group, Department of Telecommunications, ENSA, Tetouan, Morocco \\ ${ }^{3}$ Instituto ITACA, Universitat Politécnica de València, Camino de Vera, s/n, Valencia, Spain
}

\begin{abstract}
In this paper, a high power UWB monocycle generator for Non-Destructive Microwave Radar-Sensor detection is presented. The full radar system is compound with a designed UWB generator, two commercial antennas, and an oscilloscope as a receptor. The generator is composed of three essential parts-circuits, an avalanche transistor, an SRD pulse sharping and an MFN. The main idea in this work is to convert the square waveform to a driving pulse with the parameters required by the SRD pulse sharpening to obtain an output Gaussian pulse. This output pulse is converted then into a monocycle pulse. As we know, for a good range radar measurement, a high amplitude of pulse with a minimum ringing is indispensable, especially when the measurements requires many accuracies. In order to increase the pulse of the transmitter, a useful technique has been introduced; this technique consists of making the discharge path as short as possible. The applicant technique leads also to minimize the ringing in the output pulse. The measurements had been carrying out in an anechoic chamber, with the distance between 50 and $270 \mathrm{~cm}$. Good Agreements between the measured and calculated results are achieved, therefore, this system can be considered very attractive in many application fields.
\end{abstract}

\section{Introduction}

Nowadays, there are many techniques that are used as non-destructive inspection including seismic [1-2], gravity [3-4] and electromagnetic methods [5-6]. Each technique as its advantages and drawbacks, but the electromagnetic based methods seem still to be the best ones. The reason is that because they allow for higher precision in measurement and have less dependency with regards to the environmental conditions such a temperature or humidity.

The electromagnetic method is based on the design of a specific Ultra-Wide-Band (UWB) radar sensor. The interest in UWB radar sensors systems for nondestructive application has increased significantly in recent years [7-8]. Numerous of these systems work in the time domain and possess an effective frequency spectrum of around $0.1 \mathrm{GHz}$ to $10 \mathrm{GHz}$ [8]. These systems present many advantages; high precision, low power consumption, and wide-bandwidth characteristics.

The principal parts in any UWB radar sensors are UWB nanosecond pulses generators. The basis of a classical UWB short pulse generator is a driver, a pulse sharpened and a pulse-forming network. On the other hand, there are various techniques to generate a nanosecond monocycle UWB pulses. A uniplanar subnanosecond monocycle pulse generator has been reported in [9]. A UWB monocycle pulse generator with dual resistive loaded shunt stubs was presented in [10]. In addition, an UWB monocycle pulse generator with good performance has been presented in [11]. In almost all of the previous mentioned papers, the monocycle pulse amplitude was limited, and did not enable to satisfy familiar criteria of a good radar sensor capable of providing high standard accuracy which is needed in non-destructive measurement methods.

To fulfill this task, a simple technique and useful solution have been used to improve the output pulse of radar. This technique consists in making the discharge path as short as possible in the driver parts. In this manner, a minimum of ringing around $3.6 \%$ in overshoot has been obtained. However, and unlike the generator described by Zhong and Protiva [11-12], our pulse transmitter has the advantage of producing pulses with low ringing levels and reasonably high output amplitude. These two advantages are effective in order to satisfy completely the requirement headed for getting a system having the most accurate measurement pattern.

In this paper, an electromagnetic technique in term of non-destructive methods is presented. This method consists of a designed UWB radar sensor system having high measurements accuracy. In order to demonstrate the efficiency of the proposed design, and to validate the performance of the system, some measurements of range target are presented. The measurements are made with a distance between 50 and $270 \mathrm{~cm}$ in an anechoic chamber

\footnotetext{
${ }^{a}$ Corresponding author: youah@itaca.upv.es
} 
in which any disturbance of parasitic signals is avoided. The pulse generator circuit is entirely fabricated on a microstrip structure, which fulfills the characteristics of being low-cost, compact and miniaturization. Demonstration that good agreement between the measured and calculated result is achieved will be given.

\section{Radars System Description}

There are two principal configurations in the radar systems, the bi-static and the mono-static configuration; the first one happens when the modules of transmission and reception use two separated antennas, while the second one occurs when the modules of transmission and reception form a unique antenna [13]. The mono-static presents many disadvantages and needs arrangements that are technically more difficult. Therefore, in this paper, we have chosen the bi-static configuration to deal with measurements with the purpose to improve accuracy and to get more efficient outcome. In Figure 1, the whole system having bi-static configuration is presented. In order to avoid any perturbations and interferences during the measurements, the transmitting $T_{x}$ and the receiving antenna $R_{x}$ have been separated by a distance of $20 \mathrm{~cm}$

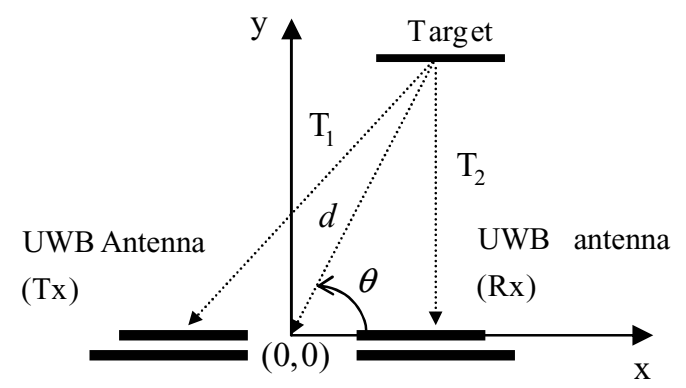

Figure 1. Bi-static system configuration of the radar.

The circuit schematic of the proposed impulse generator is shown in Figure 2. It is constructed by two principal parts: the avalanche transistor circuit and SRD pulse sharpener circuit.

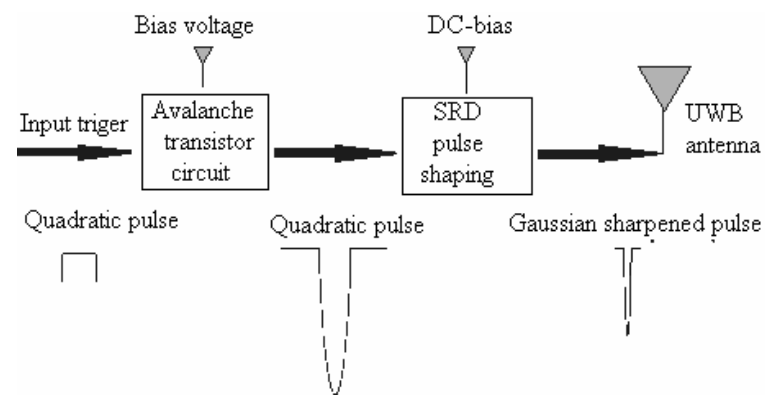

Figure 2. Circuit diagram of the proposed Gaussian pulse generator.

The first part of the generator consists of $Q, C_{1}, R_{1}, C_{2}$, and $R_{2}$ as shown in Figure 3. The details description of the generator can be found in [14].
The second part of the generator consists of an SRD sharpener and a Gaussian pulse forming the entire network as shown in Figure 4. The more detailed description can be encountered in the works presented in [14]. Furthermore, making the discharge path as short as possible, a minimum of the ringing has been obtained in the output pulse. The obtained overshoot was of the order of $3.6 \%$.
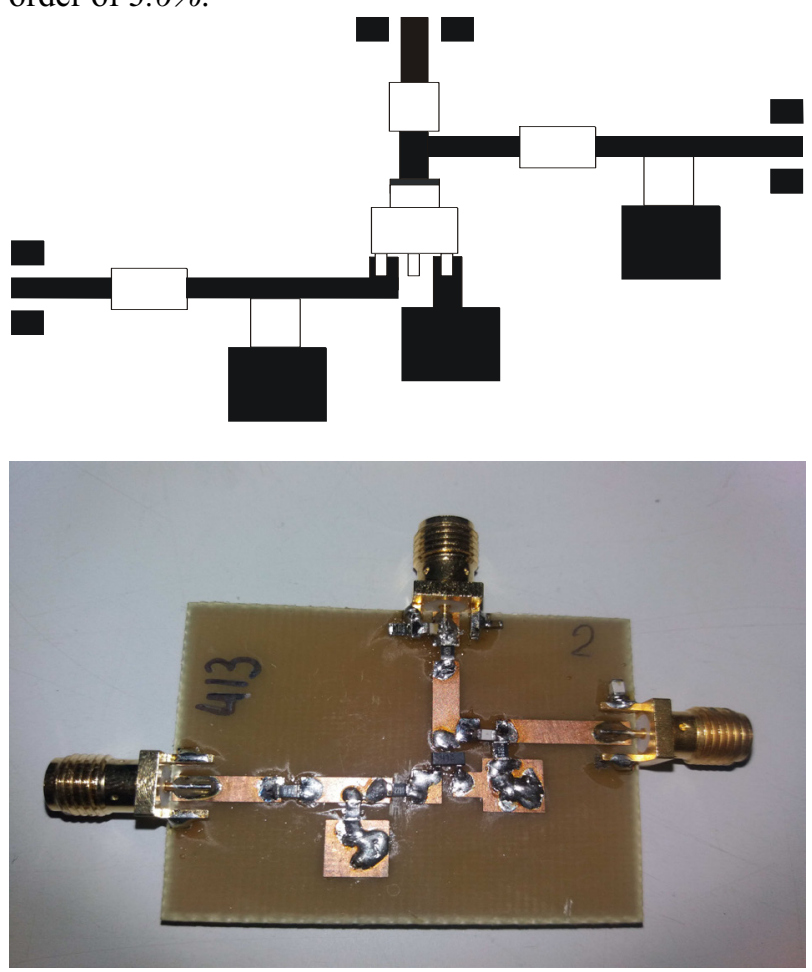

Figure 3. Layout and circuit designed for the avalanche transistor circuit.
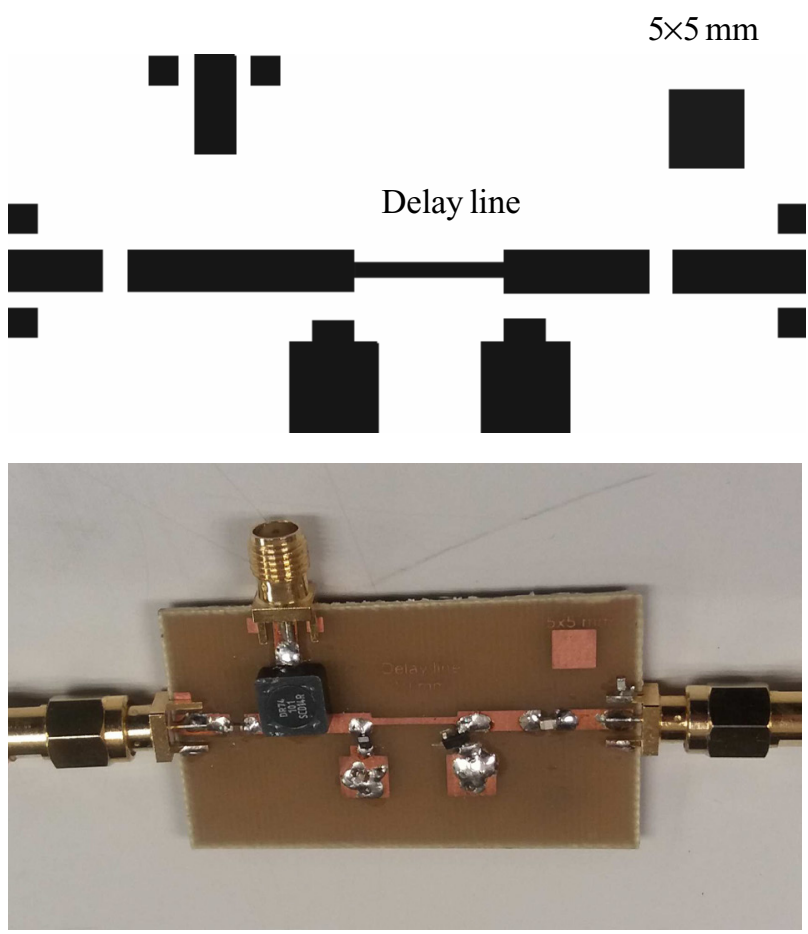

Figure 4. The designed system with SRD pulse sharpener and the corresponding equivalent Layout circuit. 


\section{Measurements}

The developed radar sensor was constructed in bi-static configuration. The measurement setup of the sensor is shown in Figure 5. The measurements of the distance range have been made with the famous Time-of-Flight principle (ToF).

In order to avoid any parasitic signal between the transmitter $\left(T_{x}\right)$ and receiver $\left(R_{x}\right)$. The separation distance was elected to be $20 \mathrm{~cm}$. Therefore high voltage pulses with amplitude of $6.4 \mathrm{~V}$ at $50 \Omega$ are generated in all measurements.

Figure 6 shows the antennas which have been used in the measurements. They are UWB commercial antennas from RFSPACE Inc. The antennas have a high gain, low VSWR and broadband response. These antennas cover a UWB from $0.6 \mathrm{GHz}$ to $6 \mathrm{GHz}$.

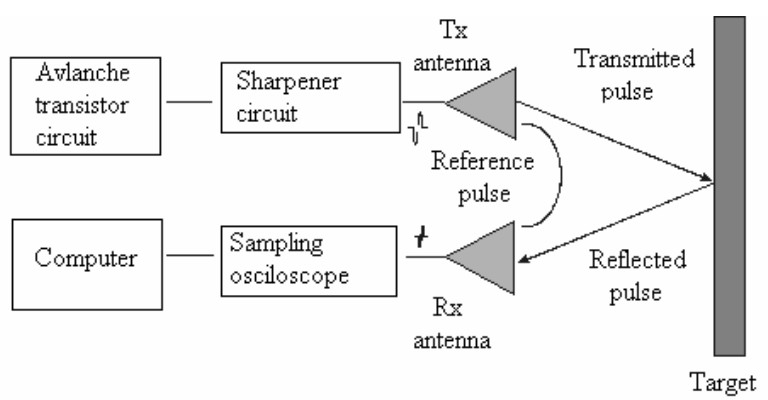

Figure 5. Measurement setup of the bi-static Radar sensor.

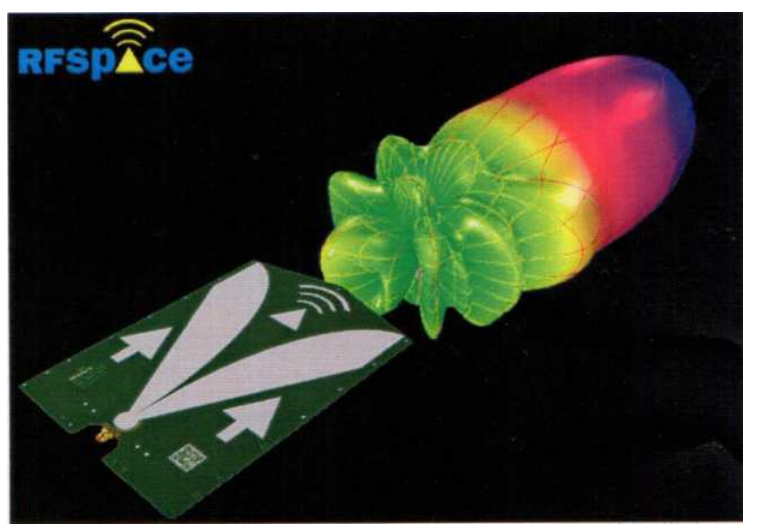

Figure 6. Photograph of the UWB antenna with the radiation pattern.

To test the efficiency of the designed radar sensor, a metal plate with a size of $30 \mathrm{~cm} \times 70 \mathrm{~cm} \times 3 \mathrm{~mm}$ has been chosen to measure the distance accuracy. For this purpose, many measurements for different distance objects ranging between $50 \mathrm{~cm}$ to $270 \mathrm{~cm}$ have been executed. The distance of the target at $50 \mathrm{~cm}$ has been fixed just to confirm the operability of the designed system prototype and their performance in short range application measurements. The measurements setup of the pulse radar sensor for the selected target distance and the photograph of UWB impulse generator are shown in Figure 7.

More improvement on the signal reception and better use of data at the reception can be taken out with a developed signal processing, but this was not the principal aim of this work. Our purpose in this paper was to report the hardware development and to demonstrate the workability of the radar sensor prototype in nondestructive measurements.
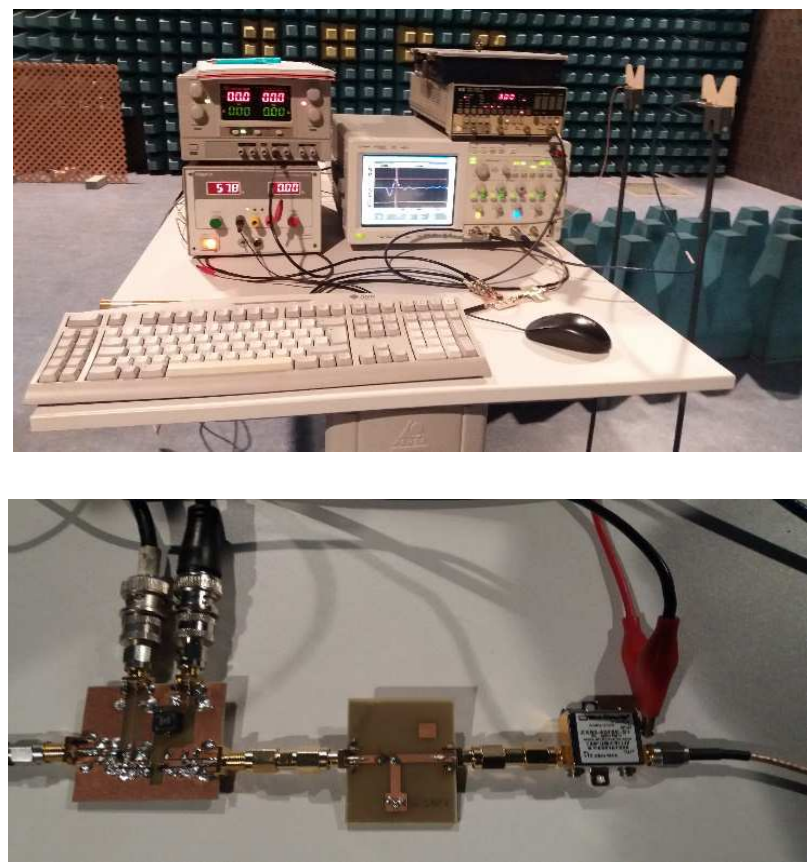

Figure 7. Photograph of measurement setup and a photograph of UWB impulse generator.

The time-domain response of the radar sensor measurement towards the metal plate located at $60 \mathrm{~cm}$ is shown in Figure 8. We observe clearly from the behavior of the curve, the presence of the reference pulse and the reflected pulse from the object; the reflected pulse was accompanied with some unwanted peaks and some ringing, this unwanted ringing occurred however after the target return is received.

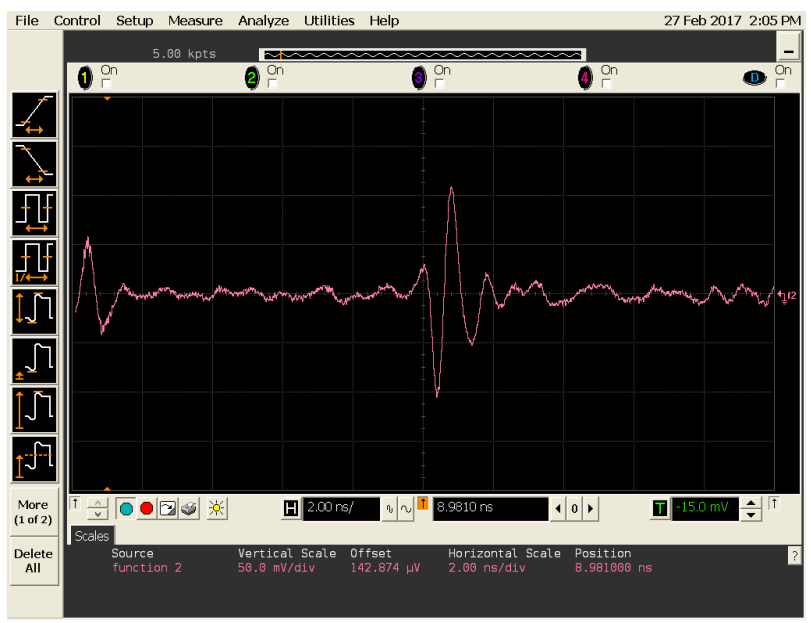

Figure 8. The time-domain response of the radar sensor measurement towards a metal plate located at $50 \mathrm{~cm}$.

\section{Conclusions}

A UWB monocycle generator for Non-Destructive Microwave Radar-Sensor detection has been presented. 
The system employs a monocycle impulse transmitter with $6.4 \mathrm{~V}$ in amplitude, a $0-6 \mathrm{GHz}$ sampling oscilloscope as a receiver, and two UWB antennas operating from $0.6 \mathrm{GHz}$ to $6 \mathrm{GHz}$. In order to eliminate the ringing in the output pulse waveform, a useful technique has been used. This technique consists of making the discharge path as short as possible. Many measurements and test have been made to confirm the performance of this system prototype. Experimental data, obtained with this first-design system and a simple measurement procedure, also agree well with the theoretical results. Good performance together with the compactness and low cost of the impulse generator make this system very attractive as a useful tool for many non-destructive measurements.

\section{References}

1. L. Guanghui, L. Yue, Y. Baojun, IEEE Transactions on Geoscience and Remote Sensing, 55 (2017)

2. X. Dijia, B. Hongxia, J. Zheng, 25th Annual International Symposium on Personal, Indoor, and Mobile Radio Communication (Washington DC, USA, 2014)

3. S. Fritz, K. Turkoglu, International Journal of Aerospace Engineering (2017)

4. O. B. Andersen, J. Maulik, IEEE International Geoscience and Remote Sensing Symposium (Quebec City, Canada, 2014)

5. Y. Yu, C.C. Chen, IEEE International Geoscience and Remote Sensing Symposium (Fort Worth, USA 2017)

6. Y. Fuse, M. Rostami, B. Gonzalez-Valdes, C.M. Rappaport, 11th European Conference on Antennas and Propagation (Paris, France, 2017)

7. P. Bernardi, R. Cicchetti, S. Pisa, E. Pittella, E. Piuzzi, O. Testa, IEEE Sensors Journal, 14 (2014)

8. X. Xu, T. Xia, V. Anbu, H. Dryver, ASCE's Journal of Engineering Mechanics, 139 (2013)

9. J.S. Lee, C. Nguyen, T. Scullion, IEEE Trans Microwave Theory Tech., 49 (2001)

10. T.G. Ma, C.J. Wu, P.K. Cheng, C.F. Chou, Microwave and Optical Technology Letters 92, 2 (2007)

11. Z. Li, X.X. Lv, Y.F. Long, T. Qin, Proc. Int. Conf. Computat. Problem-Solving (2011)

12. P. Protiva, J. Mrkvica, J. Machac, Microwave and Optical Technology Letters 52, 11 (2010)

13. A.A.H Ameri, Long-Range Ultra-Wideband Radar Sensor for Industrial Applications (Kassel University, Germany, 2012)

14. Y. Ahajjam, O. Aghzout, J.M. Catala-Rivera, F. Peñaranda Foix, A. Driouach, 5th International Conference on Multimedia Computing and Systems (Marrakech, Morocco, 2016) 